\title{
Um modelo kaleckiano com gasto público em educação *
}

\author{
Adriano Renzi ${ }^{* *}$ \\ José Gabriel Porcile Meirelles ***
}

\section{Resumo}

O artigo discute como a política fiscal, através dos gastos em educação, afeta a acumulação de capacidades e o nível de emprego. Desse modo, o gasto público pode afetar a produtividade e ter efeitos que vão além daqueles diretos sobre a demanda efetiva. $\mathrm{O}$ efeito sobre a produtividade deriva do fato de que parte do gasto público se destina à educação e ao treinamento dos trabalhadores. São discutidos os efeitos da política fiscal sobre o emprego, a produtividade e o crescimento, e as implicações de política do modelo.

Neste artigo pretende-se analisar como a política fiscal, via gastos em educação, pode afetar a acumulação de capital humano e o nível de produtividade de um país e, consequentemente, alterar o nível de emprego e catalisar o crescimento econômico. A contribuição teórica proposta advém do fato de que a questão central é incluir uma nova dimensão, ou seja, a possibilidade de que o gasto público possa contribuir para os aumentos da produtividade. Isto é, o gasto do governo tem um efeito que vai além da demanda efetiva; ele pode afetar também a produtividade do trabalho, agora não mais constante. Neste modelo admitiremos que certa parcela do gasto público seja investida em educação. Dessa forma, se estabelece um vínculo entre política fiscal, educação e produtividade. A partir daí, serão obtidos novos resultados em termos dos níveis de emprego, salário e produto, e suas implicações de política poderão ser analisadas.

Palavras-chave: Política fiscal; Produtividade; Capital humano; Educação; Crescimento.

\section{Abstract \\ Kaleckian a model with public spending on education}

This article discusses how fiscal policy, through the spending on education, affects the accumulation of capabilities and the levels of productivity. It is suggested the possibility that public spending may contribute to productivity increases, therefore having an effect that goes beyond its direct effect on effective demand. The effect on productivity comes from the investment of a share of total public spending in education and the training of workers. As a result, public spending gives rise to changes in employment levels, wages, productivity and growth, whose policy implications are analyzed.

Keywords: Fiscal policy; Productivity; Human capital; Education; Growth.

JEL E120, E240, E620, O110, O400.

* Trabalho recebido em 12 de outubro de 2011 e aprovado em 14 de abril de 2013.

** Professor da Universidade Federal da Grande Dourados (UFGD) / Professor do Centro Universitário da Grande Dourados (Unigran), Dourados, MS, Brasil. E-mail: adrenzi@ yahoo.com.br.

*** Professor Doutor da Universidade Federal da Paraná (UFPR / Pesquisador do Conselho Nacional de Desenvolvimento Científico e Tecnológico (CNPq), Curitiba, Paraná, Brasil. E-mail: porcile@ufpr.br.

Economia e Sociedade, Campinas, v. 23, n. 2 (51), p. 319-339, ago. 2014 


\section{Introdução}

Nos modelos kaleckianos, as firmas atuam em mercados com concorrência oligopolística, ou seja, elas têm poder para determinar o preço de seus produtos, os quais são considerados heterogêneos e, também, as firmas podem operar com capacidade ociosa. Como resultado dessas hipóteses iniciais, nos modelos com inspiração kaleckiana (Steindl, 1952) é possível haver uma relação positiva entre taxa de lucro, acumulação e salário real no equilíbrio de longo prazo.

$\mathrm{Na}$ vertente kaleckiana devem ser ressaltadas, pelo menos, quatro características significativas. A primeira diz respeito ao conceito de grau de monopólio, ou seja, neste conceito são sintetizados vários fatores que influenciam os preços relacionados com os custos diretos, reduzindo o poder de influência do nível de demanda agregada sobre o nível de preços. A segunda refere-se à suposição de que, enquanto houver capacidade ociosa, os custos marginais são constantes. A terceira característica diz respeito à hipótese de que há capacidade ociosa, então, o nível de utilização da capacidade produtiva é menor que a unidade. E, por último, enfatiza-se o papel da função investimento como uma função dependente do nível da capacidade ociosa e da taxa de lucro (Ibidem).

Outro aspecto a ser mencionado, que se pretende adequar à teoria kaleckiana, é o gasto do governo em educação para a acumulação de capital humano e, por conseguinte, o impacto desse capital na distribuição da renda e no crescimento econômico do país.

O presente artigo objetiva apresentar e discutir um modelo de crescimento econômico de inspiração kaleckiana. Esse modelo apresenta uma causalidade recíproca entre nível de emprego e taxa de câmbio, que são estabelecidas por meio da resolução de questões que envolvem a dinâmica do conflito distributivo entre os agentes econômicos (governo, trabalhadores e capitalista), a competitividade da economia doméstica no mercado internacional e a influência do gasto do governo em educação (de forma mais abrangente, considera-se esse efeito como a "acumulação de capital humano", mas em referência à acumulação de capacidades em termos mais amplos, com efeitos positivos no aprendizado e na absorção de tecnologia). A configuração de todos esses elementos é determinante na distribuição da renda doméstica entre os agentes econômicos.

O artigo está subdividido da seguinte forma: na primeira seção será apresentado como é determinada a participação do salário real na renda gerada pela economia e como se estabelece a relação entre conflito distributivo no mercado de trabalho, competitividade e taxa de câmbio. Na seção seguinte será revelado como a política fiscal pode afetar a distribuição da renda e o crescimento econômico. E, por fim, o artigo é concluído com a apresentação de estáticas comparativas e as conclusões do modelo. 


\section{Participação do salário real na renda}

\section{Demanda por mão de obra}

Neste modelo, assim como no modelo desenvolvido por Carlin e Soskice $(1990,2006)$, as firmas determinam a fração da renda gerada que será destinada ao pagamento dos salários dos trabalhadores $\left(W^{F}\right)$ por meio da seguinte expressão:

$$
W^{F}=\frac{W}{P}=\frac{Z A}{T},
$$

onde $P$ é o nível de preços domésticos, $Z=1-m$ é o mark-up ${ }^{1}, W$ é o salário nominal, $T=1+\tau$ é taxa de tributação sobre o salário nominal, $\tau$ é a alíquota de tributação e $A$ é a produtividade do trabalho (Carlin; Soskice, 1990, p. 141) ${ }^{2}$.

Numa economia aberta supõe-se que o nível de preços $(\bar{P})$ seja obtido por meio da relação entre o nível de preços internacional $\left(P^{*}\right)$, a taxa de câmbio nominal $(R)$ e o nível de preços domésticos $(P)$ por intermédio da seguinte expressão ${ }^{3}$ :

$$
\bar{P}=P^{\alpha}\left(P^{*} R\right)^{1-\alpha}
$$

A partir da equação 2 pode-se estabelecer qual será a parcela da renda ofertada como salário real $\left(W^{F}\right)$ pelas firmas. Isto está representado pela seguinte expressão:

$$
W^{F}=\frac{Z}{T Q^{1-\alpha}}
$$

A parcela de salário real ofertado pelas firmas $\left(W^{F}\right)$ é diretamente relacionada ao mark-up $(Z)$, inversamente relacionada à taxa de tributação $(T)$ e à taxa de câmbio real $(Q)$. Se houver uma apreciação da taxa de câmbio real, o custo da mão de obra doméstica se torna mais alto em relação à mão de obra

(1) A determinação do preço de firmas em concorrência monopolista é dada pela seguinte expressão:

$$
P=\left(\frac{1}{1-\frac{1}{\xi}}\right) \cdot C m g=\left(\frac{1}{1-\frac{1}{\xi}}\right) \cdot \frac{W}{P m g L},
$$

onde $\xi$ é o valor absoluto da elasticidade da demanda e Cmg é o custo marginal que é igual ao salário nominal dividido pelo produto marginal do trabalho (PmgL). O mark-up é representado por $\frac{1}{1-1 / \xi}=\frac{1}{1-m}$ em que quanto menor elasticidade da demanda pelo produto, maior o mark-up. Logo, $m$ representa o inverso do valor absoluto da elasticidade da demanda.

(2) Veja que um aumento em $z$ ou em $\tau$ reduz o salário real $(\boldsymbol{w})$.

(3) $\ln \bar{P}=\alpha \ln P+(1-\alpha) \ln P^{*}+(1-\alpha) \ln R \Leftrightarrow \hat{p}=\alpha \hat{p}+(1-\alpha)\left(\hat{p}^{*}+\hat{R}\right)$. 
internacional e, portanto, haverá uma redução no salário real ofertado pelas firmas ${ }^{4}$.

$$
w^{F}=z-(1-\alpha) q-\psi
$$

Pela equação 4, pode-se inferir como cada uma das variáveis se comporta ao longo do tempo. No caso, apenas o mark-up se relaciona diretamente com o salário ofertado pelas firmas, já as outras duas variáveis se relacionam inversamente com a taxa de câmbio $(q)$ e a taxa de tributação sobre o salário ofertado $(\psi)^{5}$.

\section{Oferta de mão de obra}

O trabalho de Lewis (1986) examina a relevância dos sindicatos para os ganhos com barganha salarial. No período 1967-1979 as estimativas indicam que os trabalhadores americanos com vínculos sindicais recebiam em média $15 \%$ a mais que os trabalhadores sem nenhum vínculo sindical. Os trabalhadores sindicalizados do Reino Unido receberam em torno de 8 a 10\% (Stewart, 1983) a mais que os outros trabalhadores sem vínculos.

A evidência empírica apoia o fato de que a densidade sindical da indústria é positivamente relacionada com os ganhos dos trabalhadores sindicalizados e não sindicalizados. Adicionalmente, o crescimento na densidade sindical da indústria, ou seja, a proteção e organização (coverage) sindical induzem a maiores salários médios na firma (Layard; Nickell; Jackman, 1991, p. 196-197) ${ }^{6}$. Embora a análise feita não esclareça todas as nuances de como o poder de barganha dos sindicatos afeta o mark-up, Sen e Dutt (1995) confirmam a conjectura de Kalecki, ou seja, o poder de barganha pode afetar o mark-up utilizando uma estrutura de otimização envolvendo equilíbrio de Nash.

O salário real ofertado pelas firmas nem sempre é igual ao salário real desejado pelos trabalhadores representados pelos sindicatos. Em geral, os sindicatos demandam uma parcela da renda gerada $\left(w^{d}\right)$ levando em consideração a influência do nível de emprego $(n)$, como apresentado pela equação abaixo:

(4) Onde Q é a taxa de câmbio real da economia.

$$
Q=\frac{P * R}{P} w^{F}=\frac{W}{\bar{P}} \frac{L}{Y}=\frac{P Z A}{T P^{\alpha}\left(P^{*} R\right)^{1-\alpha}} \frac{1}{A}=\frac{P^{1-\alpha}}{\left(P^{*} R\right)^{1-\alpha}} \frac{Z}{T} \Rightarrow w^{F}=\frac{Z Q^{\alpha-1}}{T}
$$

(5) Lembrando que $Z=1-m$ e $m$ representa a elasticidade da demanda pelo produto, um aumento em $m$ provoca uma redução no mark-up e, portanto, uma redução no salário real ofertado $(W F)$. Assim, fazendo as transformações algébricas, chega-se à equação de salário real ofertado ao longo do tempo.

$$
\ln W^{F}=w^{F}=\ln Z-(1-\alpha) \ln Q-\ln T \text {, onde } \ln W^{F}=w^{F}, \ln Z=z, \ln Q=q \text { e } \ln T=\psi .
$$

(6) Para mais detalhes sobre o adicional salarial (mark-up union) proporcionado pela proteção sindical na indústria britânica, ver Layard et al. (1991). 


$$
w^{d}=n \gamma,
$$

onde a parcela da renda demandada como salário real pelos sindicatos $\left(w^{d}\right)$ depende diretamente da velocidade de resposta ( $\gamma$ ) do nível de emprego $(n)^{7}$.

Se, por hipótese, o mark-up se mantém constante, a taxa de crescimento do salário real $(\hat{w})$ é igual à inflação $(\hat{p})$ mais a taxa de crescimento da produtividade do trabalho $(\hat{a})$ e o resultado da disputa por uma maior parcela da renda real como salário entre sindicatos e firmas $\beta\left(w^{d}-w^{F}\right)$, com $0<\beta<1$, sendo $\beta$ o coeficiente de velocidade de ajustamento no mercado de trabalho. Como em Dutt (1990), será suposto que as variações no salário nominal dependem do hiato entre o salário real desejado pelo sindicato $\left(w^{d}\right)$ e o salário real ofertado pelas firmas $\left(w^{F}\right)$.

O ajustamento dos salários e preços resulta da tentativa de firmas e empregados realizarem suas metas desejadas no processo de barganha. $\mathrm{O}$ maior salário desejado pelos sindicatos depende da taxa de variação do emprego. Portanto, em equilíbrio, a participação dos trabalhadores e das firmas na renda e a taxa de inflação podem ser interpretadas como o equilíbrio das forças sociais (Cassetti, 2003, p. 462).

$$
\hat{w}=\hat{p}+\hat{a}+\beta\left(w^{d}-w^{F}\right) .
$$

Substituindo as equações 4 e 5 na 6 encontramos a equação de dinâmica temporal da taxa de câmbio real ${ }^{8}$ :

$$
\dot{q}=\frac{\beta}{(1-\alpha)}[z-(1-\alpha) q-\psi-n \gamma]
$$

No steady state a taxa de câmbio de equilíbrio $\left(q^{*}\right)$ é afetada diretamente pelo mark-up $(z)$ e inversamente pela taxa de tributação do salário ofertado $(\psi)$ e pelo nível de emprego $(n)$. Desse modo, um aumento no mark-up das firmas nacionais ou uma queda na taxa de tributação do salário ofertado ou no nível de emprego da economia doméstica gera uma desvalorização da taxa de câmbio nacional. A taxa de câmbio de equilíbrio é representada pela seguinte expressão:

$$
q^{*}=\frac{1}{(1-\alpha)}[z-\psi-n \gamma]
$$

Nesta seção determinamos a participação do salário real na renda gerada pela economia e concluímos, por meio da equação 8 , como se estabelece a relação entre conflito distributivo no mercado de trabalho, competitividade e taxa de câmbio. Portanto, o conjunto de equações apresentado anteriormente leva em conta

$$
\begin{aligned}
& \text { (7) O salário demandado pelos sindicatos é expresso por } W^{d}=N^{\gamma} \text { ou } \ln W^{d}=w^{d}=\gamma \ln N=n \gamma \text {. } \\
& \text { (8) } \dot{q}=\hat{p}-\hat{p}^{*}+\hat{R} \text {. }
\end{aligned}
$$


as condições do mercado de trabalho e a importância da taxa de câmbio para estabelecer o equilíbrio macroeconômico da economia doméstica. Na próxima seção discutiremos como a política fiscal pode ser uma alternativa para afetar a distribuição da renda e o crescimento econômico.

\section{Política fiscal, capital humano e emprego}

O gasto do governo em educação potencializa a capacidade dos indivíduos de aprender e, consequentemente, de poderem vir a ser mais criativos. A maior velocidade na acumulação de conhecimento permite o maior efeito positivo do learning by doing sobre a produtividade do trabalho. Neste modelo, assim como no de Mankiw, Romer e Weil (1992) $)^{9}$ o capital humano $(H)$ é a quantidade de trabalho produtivo ofertado pelos trabalhadores. Em outras palavras, é a contribuição total dos trabalhadores de diferentes níveis de habilidade (skills) para a produção. Os níveis de habilidade incluem as habilidades inerentes ao indivíduo e as habilidades adquiridas ao longo do tempo.

Ao descrever e, posteriormente, inserir a relação entre o capital humano e a produtividade do trabalho neste modelo de uma economia em desenvolvimento, a hipótese inicial aqui será a de que a acumulação de capital humano $(\dot{H})$ é financiada inteiramente pelo governo. No caso, o governo investe uma parcela $(g)$ desses impostos em educação ${ }^{10}$ :

$$
\dot{H}=g \tau Y .
$$

Outra suposição é a de que a renda (ou produto) gerada $(Y)$ equivale a uma combinação do emprego $(N)$, da produtividade do trabalho $(A)$ e da força de trabalho disponível $(L)$, dada por $(Y=N A L)$. Além disso, a produtividade do trabalho $(A)$ é proporcional ao estoque de capital humano por pessoa $(A=H / L)$, assim tem-se:

$$
Y=N H
$$

A partir da equação 10 , pode-se inferir que o produto se relaciona diretamente com o nível de emprego e com o estoque de capital humano. Inserindo a equação 10 na 9 e fazendo as transformações algébricas necessárias encontra-se a

(9) O modelo de Mankiw, Romer e Weil (1992) contribuiu empiricamente para a discussão tentando medir e verificar os diferentes canais pelos quais o capital humano atua sobre o crescimento econômico.

(10) Por sua vez, Lucas (1988) argumenta que a acumulação de capital humano ocorre quando os indivíduos alocam uma parte de seu tempo de trabalho para a sua própria educação.

$\dot{H}=\phi u H-\delta H$ onde $\delta, 0<\delta<1$, é a depreciação do capital humano e $\phi, 0<\phi<1$, é o nível de eficiência da educação. 
taxa de crescimento de uma economia em desenvolvimento $(\hat{H})$ com referência à acumulação de capital humano ${ }^{11}$.

$$
\hat{H}=g\left(e^{\psi}-1\right) e^{n}
$$

Se os preços relativos não variam no longo prazo, pode-se considerar que esse modelo com restrição no balanço de pagamentos utiliza a Lei de Thirlwall (1979) para explicar o crescimento econômico $(\hat{Y})$ da economia, como apresentado pela seguinte expressão ${ }^{12}$ :

$$
\hat{Y}=\frac{x}{\pi}
$$

A Lei de Thirlwall diz que a taxa de crescimento que um país pode sustentar depende do crescimento do resto do mundo e da elasticidade da renda das importações $(\pi)$ e das exportações $(x)$. A razão entre as elasticidades reflete o grau de competitividade dos produtos nacionais no mercado internacional e o grau de competitividade do mercado doméstico; ambos determinam a especialização estrutural do país e o grau de diferenciação do produto (Ledesma, 2002, p. 10).

$$
\hat{Y}=\frac{\hat{Y}^{*}+\varepsilon(\hat{H}-\hat{\rho})}{\pi}
$$

A evolução do crescimento do produto nacional depende da evolução das exportações $(x)$ e esta, por sua vez, depende do crescimento da renda internacional $\left(\hat{Y}^{*}\right)$ mais o resultado da diferença entre a taxa de crescimento da produtividade da economia em desenvolvimento, representada pela acumulação de capital humano $(\hat{H})$, e a taxa de crescimento da produtividade no resto do mundo $(\hat{\rho})$. O coeficiente $\varepsilon, 0<\varepsilon<1$, reflete velocidade de ajustamento entre a dinâmica tecnológica interna e a externa. Esse coeficiente reflete a importância do diferencial de produtividade sobre a taxa de crescimento da economia em desenvolvimento. O modelo considera que a taxa de câmbio nominal é fixa e não desempenha nenhum papel no modelo.

$$
\begin{aligned}
& \text { (11) } T=1+\tau \Leftrightarrow \psi=\ln (1+\tau) \Leftrightarrow e^{\psi}=1+\tau \Leftrightarrow \tau=e^{\psi}-1 \\
& N=e^{n} \Leftrightarrow n=\ln N \\
& \dot{H}=g\left(e^{\psi}-1\right) Y=g\left(e^{\psi}-1\right) N L A \Leftrightarrow \dot{H}=g\left(e^{\psi}-1\right) e^{n} H \Leftrightarrow \frac{\dot{H}}{H}=g\left(e^{\psi}-1\right) e^{n}
\end{aligned}
$$

(12) Há uma vasta literatura teórica e empírica que demonstra a relevância da Lei de Thirlwall para explicar por que as taxas de crescimento dos países diferem entre si (McCombie; Thirlwall, 1994; 1999).

$$
y=\ln Y \Rightarrow \frac{\partial y}{\partial t}=\dot{y} \Rightarrow \frac{\dot{Y}}{Y}=\hat{Y}
$$


A evolução da taxa de emprego no tempo pode ser escrita como ${ }^{13}$ :

$$
\hat{N}=\hat{Y}-\hat{H}
$$

Usando as equações 11,12 e 13 na equação 14, formaliza-se a expressão que representa a dinâmica de crescimento do emprego ${ }^{14}$ :

$$
\hat{N}=\frac{\hat{Y}^{*}+g(\varepsilon-\pi)\left(e^{\psi}-1\right) e^{n}-\varepsilon \rho}{\pi}
$$

A solução anterior supõe que a taxa de crescimento da renda internacional deve ser maior que a taxa de crescimento da produtividade no resto do mundo $\left(\hat{Y}^{*}>\rho\right)$ e que a elasticidade da renda das importações $(\pi)$ deve ser maior que a velocidade de ajustamento entre a dinâmica tecnológica interna e a externa $(\varepsilon)$ $(\pi>\varepsilon)$. O sistema resultante tem duas equações diferenciais:

$$
\begin{aligned}
& \dot{q}=\frac{\beta}{1-\alpha}[z-(1-\alpha) q-\psi(\tau)-n \gamma] \\
& \dot{n}=\frac{\hat{Y}^{*}+g(\varepsilon-\pi)\left(e^{\psi}-1\right) e^{n}-\varepsilon \rho}{\pi}
\end{aligned}
$$

A matriz jacobiana desse modelo é dada por:

$$
|J|=\left|\begin{array}{ll}
\frac{\partial \dot{q}}{\partial q} & \frac{\partial \dot{q}}{\partial n} \\
\frac{\partial \dot{n}}{\partial q} & \frac{\partial \dot{n}}{\partial n}
\end{array}\right|=\left|\begin{array}{cc}
-\beta & -\frac{\beta \gamma}{1-\alpha} \\
0 & \frac{g(\varepsilon-\pi)\left(e^{\psi}-1\right) e^{n}}{\pi}
\end{array}\right|
$$

A condição necessária e suficiente para a estabilidade global do sistema é dada por:

$$
\operatorname{tr}|J|<0 \Rightarrow(-\beta)+\frac{g(\varepsilon-\pi)\left(e^{\psi}-1\right) e^{n}}{\pi}<0
$$

(13) $A=\frac{H}{L} \Rightarrow \hat{A}=\hat{H}-\hat{L}$ para $\hat{L}=0 \Rightarrow \hat{A}=\hat{H}$

$N=\frac{Y}{A} \Rightarrow \hat{N}=\hat{Y}-\hat{A} \Rightarrow \hat{N}=\hat{Y}-\hat{H}$

(14) $\hat{Y}-\hat{H}=\hat{N}=\frac{\hat{Y}^{*}+\mathcal{E}(\hat{H}-\rho)-\hat{H} \pi}{\pi}$

$\hat{Y}-\hat{H}=\hat{N}=\frac{\hat{Y}^{*}+\hat{H}(\varepsilon-\pi)-\varepsilon \rho}{\pi}$ 


$$
\operatorname{Det}|J|>0 \Rightarrow(-\beta)\left[\frac{g(\varepsilon-\pi)\left(e^{\psi}-1\right) e^{n}}{\pi}\right]>0 .
$$

A equação característica deste modelo é dada pela seguinte expressão:

$$
\lambda^{2}-\operatorname{tr}\left[(-\beta)+\frac{g(\varepsilon-\pi)\left(e^{\psi}-1\right) e^{n}}{\pi}\right] \lambda+(-\beta)\left[\frac{g(\varepsilon-\pi)\left(e^{\psi}-1\right) e^{n}}{\pi}\right] \lambda=0
$$

Contudo, a convergência para o ponto de equilíbrio pode assumir duas trajetórias distintas: uma espiral ou um nódulo. Esta trajetória depende do lócus de $(\operatorname{tr}|J|, \operatorname{Det}|J|)$ cuja forma da parábola é definida por:

$$
(\operatorname{tr}|J|)^{2}-4(\operatorname{Det}|J|)=0
$$

Como $\operatorname{tr}(A)^{2}>4 \operatorname{Det}|A|$, tem-se que as raízes são reais e distintas. Como $\varepsilon<\pi, \quad \operatorname{logo} \beta g(\varepsilon-\pi) \tau N<0$, as raízes serão reais e distintas se $\left.\beta^{2}+\left[g(\varepsilon-\pi)\left(e^{\psi}-1\right) e^{n}\right]^{2}>2 \beta\left[g(\pi-\varepsilon)\left(e^{\psi}-1\right) e^{n}\right]\right]^{15}$.

A condição necessária e suficiente para que o ponto de equilíbrio $(q, n)$ seja assintoticamente estável é a de que ambos os autovalores da matriz $\mathbf{J}$ sejam negativos. Os autovalores $\left(\xi_{1}, \xi_{2}\right)$ da matriz J são dados por:

$$
\begin{aligned}
& \xi_{1}=\frac{-\left(\beta-g(\varepsilon-\pi)\left(e^{\psi}-1\right) e^{n}\right)+\sqrt{\left(\beta-g(\varepsilon-\pi)\left(e^{\psi}-1\right) e^{n}\right)^{2}+4 \beta g(\varepsilon-\pi)\left(e^{\psi}-1\right) e^{n}}}{2} \\
& \xi_{1}=\frac{-\beta+g(\varepsilon-\pi)\left(e^{\psi}-1\right) e^{n}+\sqrt{\left(\beta-g(\varepsilon-\pi)\left(e^{\psi}-1\right) e^{n}\right)^{2}+4 \beta g(\varepsilon-\pi)\left(e^{\psi}-1\right) e^{n}}}{2}
\end{aligned}
$$

Como $-\beta+g(\varepsilon-\pi)\left(e^{\psi}-1\right) e^{n}<0$, para que $\xi_{1}<0$, então será necessário que:

$$
\begin{gathered}
-\beta+g(\varepsilon-\pi)\left(e^{\psi}-1\right) e^{n}<-\sqrt{\left(\beta-g(\varepsilon-\pi)\left(e^{\psi}-1\right) e^{n}\right)^{2}+4 \beta g(\varepsilon-\pi)\left(e^{\psi}-1\right) e^{n}} . \\
\xi_{2}=\frac{-\beta+g(\varepsilon-\pi)\left(e^{\psi}-1\right) e^{n}-\sqrt{\left(\beta-g(\varepsilon-\pi)\left(e^{\psi}-1\right) e^{n}\right)^{2}+4 \beta g(\varepsilon-\pi)\left(e^{\psi}-1\right) e^{n}}}{2}
\end{gathered}
$$

$\xi_{2}<0$, visto que $-\beta+g(\varepsilon-\pi)\left(e^{\psi}-1\right) e^{n}<0$. Desse modo, os autovalores deste modelo são reais e distintos $\left(\xi_{1}, \xi_{2}\right)$.

Consequentemente, como os autovalores deste modelo são reais e distintos $\left(\xi_{1}, \xi_{2}\right)$, pela Figura 1, concluímos que o ponto de equilíbrio entre a taxa de câmbio real e o nível de emprego ao longo do tempo $(q, n)$ seja assintoticamente estável.

(15) Para mais detalhes sobre as condições de estabilidade ver Shone (1997, p. 126-131) ou Takayama (1993, p. 385-389). 
Na próxima seção demonstraremos o equilíbrio, a dinâmica comparativa e será feita uma análise de cada uma das variáveis participantes do modelo.

Figura 1

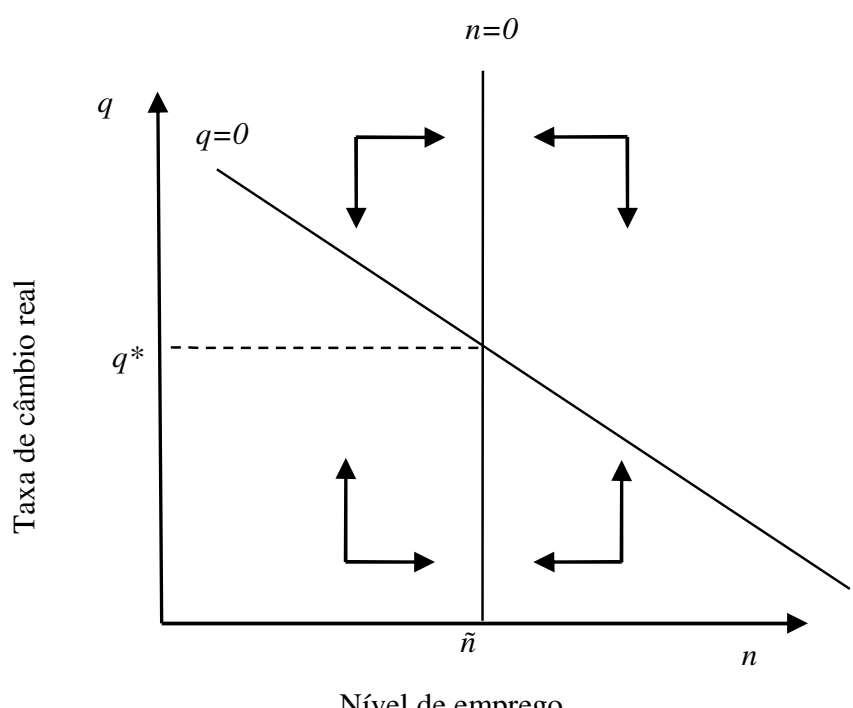

Nível de emprego

\section{Dinâmica comparativa}

A partir do sistema de equações, logo abaixo, em que é apresentada a dinâmica entre a taxa de crescimento do câmbio e a taxa de crescimento do emprego, no steady state $\dot{n}=\dot{q}=0$, tem-se:

$$
\begin{aligned}
& \dot{q}=\frac{\beta}{1-\alpha}[z-(1-\alpha) q-\psi(\tau)-n \gamma]=0 \\
& \dot{n}=\frac{\hat{Y}^{*}+g(\varepsilon-\pi)\left(e^{\psi}-1\right) e^{n}-\varepsilon \rho}{\pi}=0
\end{aligned}
$$

O valor de equilíbrio para o nível de emprego $(\tilde{n})$ é dado pela seguinte equação:

$$
\tilde{n}=\ln \left[\frac{\varepsilon \rho-\hat{Y}^{*}}{g(\varepsilon-\pi)\left(e^{\psi}-1\right)}\right]
$$

Pela equação 18 nota-se que o nível de emprego não depende da taxa de câmbio. Substituindo o nível de emprego de equilíbrio $(\widetilde{n})$ na outra equação do sistema 16, chega-se à taxa de câmbio de equilíbrio, que é dada por: 


$$
\tilde{q}=\frac{1}{1-\alpha}\left\{z-\psi-\gamma \ln \left[\frac{\left(\varepsilon \rho-\hat{Y}^{*}\right)}{g(\varepsilon-\pi)\left(e^{\psi}-1\right)}\right]\right\}
$$

Pela equação 19 , supondo que no longo prazo a taxa de crescimento da população se mantenha constante $(\hat{N}=0)$, resulta a igualdade entre a taxa de crescimento da renda e a taxa de crescimento da acumulação de capital humano, ou seja, $\hat{Y}=\hat{H}$. Por conseguinte, tem-se a evolução no tempo do crescimento da renda ou produto:

$$
\hat{Y}=\frac{\varepsilon \rho-\hat{Y} *}{\varepsilon-\pi}
$$

Portanto, as equações 18,19 e 20 de equilíbrio são dadas por $^{16}$ :

$$
\begin{aligned}
& \tilde{n}=\ln \left[\frac{\varepsilon \rho-\hat{Y}^{*}}{g(\varepsilon-\pi)\left(e^{\psi}-1\right)}\right]=\ln \left[\frac{\varepsilon \rho-\hat{Y}^{*}}{g \tau(\varepsilon-\pi)}\right], \text { onde } \hat{Y}^{*}>\varepsilon \rho \text { e } \pi>\varepsilon . \\
& \tilde{q}=\frac{1}{1-\alpha}\left\{z-\psi-\gamma \ln \left[\frac{\left(\varepsilon \rho-\hat{Y}^{*}\right)}{g(\varepsilon-\pi)\left(e^{\psi}-1\right)}\right]\right\} \\
& \hat{Y}=\frac{\varepsilon \rho-\hat{Y}^{*}}{(\varepsilon-\pi)}
\end{aligned}
$$

A partir das três equações $(18,19$ e 20) de equilíbrio apresentadas, pode-se estimar como a variação dos parâmetros deste modelo afetará o nível de emprego, a taxa de câmbio e a taxa de crescimento da renda doméstica. A Tabela 1, logo abaixo, apresenta todos os resultados da dinâmica comparativa.

A Tabela 2 foi construída de acordo com os resultados encontrados na Tabela 1. Lembrando que este modelo supõe existir capacidade ociosa, a política de aumento no gasto do governo em educação (g) afeta o nível de emprego $\left(\tilde{n}_{1} \leftarrow \tilde{n}\right)$ negativamente e a taxa de câmbio $\left(\tilde{q} \rightarrow \tilde{q}_{1}\right)$ positivamente (passando do ponto de equilíbrio a para b, Figura 2), mas gera efeitos positivos sobre a taxa de crescimento da renda doméstica $(\hat{Y})$.

(16) Lembrando que $\alpha, \beta, \pi, \rho, \tau, \gamma$ e $\varepsilon$ são números entre 0 e 1 . 
Tabela 1

\begin{tabular}{|c|c|c|c|}
\hline \multirow{2}{*}{$\begin{array}{l}\text { Parâ- } \\
\text { metros }\end{array}$} & \multicolumn{3}{|c|}{ Variáveis Endógenas } \\
\hline & $\tilde{n}$ & $\tilde{q}$ & $\hat{Y}$ \\
\hline$g$ & $\frac{\partial \tilde{n}}{\partial g}=-\frac{1}{g}<0$ & $\frac{\partial \tilde{q}}{\partial g}=\left(\frac{\gamma}{1-\alpha}\right) \frac{1}{g}>0$ & $\begin{array}{c}\frac{\partial \hat{Y}}{\partial g}=\frac{\varepsilon \rho-\hat{Y}^{*}}{g(\varepsilon-\pi)}\left(1-\frac{1}{g}\right)>0, \text { se } \hat{Y}^{*}<\varepsilon \rho \mathrm{e} \\
\varepsilon>\pi\end{array}$ \\
\hline$\tau$ & $\frac{\partial \tilde{n}}{\partial \tau}=-\frac{1}{\tau}<0$ & $\begin{array}{l}\frac{\partial \tilde{q}}{\partial \tau}=\left(\frac{1}{1-\alpha}\right)\left(\frac{\gamma}{\tau}-\frac{1}{1+\tau}\right)>0, \text { se } \gamma>\frac{\tau}{1+\tau} . \\
\frac{\partial \tilde{q}}{\partial \tau}=\left(\frac{1}{1-\alpha}\right)\left(\frac{\gamma}{\tau}-\frac{1}{1+\tau}\right)<0, \text { se } \gamma<\frac{\tau}{1+\tau} .\end{array}$ & $\begin{array}{c}\frac{\partial \hat{Y}}{\partial \tau}=\frac{\varepsilon \rho-\hat{Y}^{*}}{g(\varepsilon-\pi)}\left(1-\frac{1}{\tau}\right)>0, \text { se } \hat{Y}^{*}<\varepsilon \rho \mathrm{e} \\
\varepsilon>\pi\end{array}$ \\
\hline$\pi$ & $\frac{\partial \tilde{n}}{\partial \pi}=\frac{1}{\varepsilon-\pi}<0$ & $\frac{\partial \tilde{q}}{\partial \pi}=\left(\frac{-\gamma}{1-\alpha}\right)\left(\frac{1}{\varepsilon-\pi}\right)>0$, se $\pi>\varepsilon$. & $\frac{\partial \hat{Y}}{\partial \pi}=\frac{\hat{Y} *-\varepsilon \rho}{(\varepsilon-\pi)^{2}}<0$, se $\hat{Y}^{*}>\varepsilon \rho$ \\
\hline$\rho$ & $\frac{\partial \tilde{n}}{\partial \rho}=\frac{\varepsilon}{\varepsilon \rho-\hat{Y}^{*}}<0$ & $\frac{\partial \tilde{q}}{\partial \rho}=-\left(\frac{1}{1-\alpha}\right)\left(\frac{\gamma \varepsilon}{\varepsilon \rho-\hat{Y}^{*}}\right)>0$, se $\hat{Y}^{*}>\varepsilon \rho$ & $\frac{\partial \hat{Y}}{\partial \rho}=\frac{\varepsilon}{\varepsilon-\pi}<0$, se $\pi>\varepsilon$ \\
\hline$\varepsilon$ & $\begin{array}{c}\frac{\partial \tilde{n}}{\partial \varepsilon}=\frac{\rho}{\varepsilon \rho-\hat{Y}^{*}}-\frac{1}{\varepsilon-\pi}>0, \text { se } \hat{Y}^{*}>\varepsilon \rho, \\
\pi>\varepsilon \text { e } \hat{Y}^{*}>\pi \rho \\
\frac{\partial \tilde{n}}{\partial \varepsilon}=\frac{\rho}{\varepsilon \rho-\hat{Y}^{*}}-\frac{1}{\varepsilon-\pi}<0, \text { se } \hat{Y}^{*}>\varepsilon \rho, \\
\pi>\varepsilon \text { e } \hat{Y}^{*}<\pi \rho\end{array}$ & $\begin{array}{c}\frac{\partial \tilde{q}}{\partial \varepsilon}=\left(\frac{-\gamma}{1-\alpha}\right)\left[\frac{\rho}{\varepsilon \rho-\hat{Y}^{*}}-\frac{1}{\varepsilon-\pi}\right]<0, \text { se } \\
\hat{Y}^{*}>\varepsilon \rho, \pi>\varepsilon \text { e } \hat{Y}^{*}>\pi \rho \\
\frac{\partial \tilde{q}}{\partial \varepsilon}=\left(\frac{-\gamma}{1-\alpha}\right)\left[\frac{\rho}{\varepsilon \rho-\hat{Y}^{*}}-\frac{1}{\varepsilon-\pi}\right]>0, \text { se } \hat{Y}^{*}>\varepsilon \rho, \\
\pi>\varepsilon \text { e } \hat{Y}^{*}<\pi \rho .\end{array}$ & $\begin{array}{c}\frac{\partial \hat{Y}}{\partial \varepsilon}=\frac{\varepsilon^{2}+\hat{Y} *-\varepsilon(\rho+\pi)}{(\varepsilon-\pi)^{2}}>0, \text { se } \\
\varepsilon^{2}+\hat{Y}^{*}>\varepsilon(\rho+\pi) \text { e } \pi>\varepsilon \\
, \text { se }\end{array}$ \\
\hline
\end{tabular}

Continua... 


\begin{tabular}{|c|c|c|c|}
\hline$z$ & $\frac{\partial \tilde{n}}{\partial z}<0$ & $\frac{\partial \tilde{q}}{\partial z}=\frac{1}{1-\alpha}>0$ & $\frac{\partial \hat{Y}}{\partial z}<0$ \\
\hline$\hat{Y} *$ & $\frac{\partial \tilde{n}}{\partial \hat{Y}^{*}}=-\frac{1}{\varepsilon \rho-\hat{Y}^{*}}>0$ & $\frac{\partial \tilde{q}}{\partial \hat{Y}^{*}}=\left(\frac{1}{1-\alpha}\right)\left(\frac{\gamma}{\varepsilon \rho-\hat{Y}^{*}}\right)<0$, se $\hat{Y}^{*}>\varepsilon \rho$ & $\frac{\partial \hat{Y}}{\partial \hat{Y}^{*}}=\frac{-1}{\varepsilon-\pi^{*}}>0$, se $\pi>\varepsilon$ \\
\hline$\gamma$ & $\begin{array}{c}\frac{\partial \tilde{n}}{\partial \gamma}=-\frac{1}{\gamma^{2}}[z-\psi-q(1-\alpha)]<0, \text { se } \\
z>\psi+q(1-\alpha) \\
\frac{\partial \tilde{n}}{\partial \gamma}=-\frac{1}{\gamma^{2}}[z-\psi-q(1-\alpha)]>0, \text { se } \\
z<\psi+q(1-\alpha)\end{array}$ & $\begin{array}{c}\frac{\partial \tilde{q}}{\partial \gamma}=-\left(\frac{1}{1-\alpha}\right)\left[\ln \left(\frac{\varepsilon \rho-\hat{Y}^{*}}{g \tau(\varepsilon-\pi)}\right)\right]<0, \text { se } \\
\hat{Y}^{*}>\varepsilon \rho \text { e } \pi>\varepsilon\end{array}$ & $\begin{array}{c}\frac{\partial \hat{Y}}{\partial \gamma}=g \tau\left(\frac{\partial \tilde{n}}{\partial \gamma}\right)=-\frac{g \tau}{\gamma^{2}}[z-\psi-q(1-\alpha)]<0 \\
, \text { se } z>\psi+q(1-\alpha) \\
\frac{\partial \hat{Y}}{\partial \gamma}=g \tau\left(\frac{\partial \tilde{n}}{\partial \gamma}\right)=-\frac{g \tau}{\gamma^{2}}[z-\psi-q(1-\alpha)]>0 \\
, \text { se } z<\psi+q(1-\alpha)\end{array}$ \\
\hline
\end{tabular}

Tabela 2

\begin{tabular}{|c|c|c|c|c|c|c|c|c|}
\hline \multirow{2}{*}{ EQUILÍbRIOS } & \multicolumn{10}{|c|}{ PARAMETROS } \\
\cline { 2 - 9 } & $\mathbf{g}$ & $\tau$ & $\pi$ & $\rho$ & $\varepsilon$ & $\mathbf{z}$ & $\hat{\gamma} *$ & $\gamma$ \\
\hline$\tilde{n}$ & - & - & - & - & $(-) /(+)$ & - & + & $(-) /(+)$ \\
\hline$\tilde{q}$ & + & $(-) /(+)$ & + & + & $(+) /(-)$ & + & - & - \\
\hline$\hat{Y}$ & + & + & - & - & $(-) /(+)$ & - & + & $(-) /(+)$ \\
\hline
\end{tabular}


Figura 2

Aumento no gasto do governo em educação

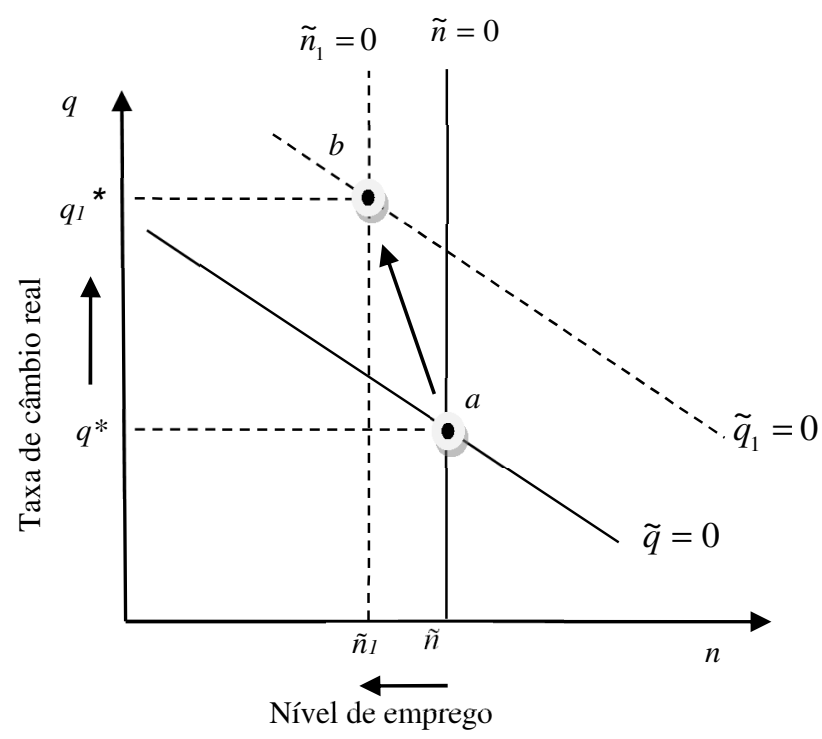

Por hipótese do modelo $(\pi>\mathcal{E})$, o resultado disso é que o efeito negativo sobre o nível de emprego, decorrente do aumento de $g$, eleva a diferença entre a elasticidade-renda das exportações $(\pi)$ e o efeito da intensidade da resposta das exportações à dinâmica da produtividade interna e externa $(\varepsilon)$ (ver equação 18 ). Já o efeito positivo sobre a taxa de câmbio é explicado pela relação positiva entre a acumulação de capital humano e a produtividade do trabalho, ou seja, o aumento da produtividade do trabalho permite a produção de uma maior quantidade de produto com a mesma quantidade de trabalho.

O ganho de produtividade reduz o custo de produção doméstico, resultado similar a uma desvalorização da taxa de câmbio $(\tilde{q})$. Esse efeito sobre o crescimento da produtividade do trabalho é crucial para explicar a relação entre redução no nível de emprego e aumento na taxa de crescimento da renda doméstica. Pode-se concluir disso que, se houver uma política permanente de gastos em educação, mantendo todos os outros parâmetros constantes, haverá uma redução contínua na taxa de emprego.

No entanto, há, claramente, um limite tanto em termos dos aumentos no gasto em educação como da estabilidade de um sistema com uma taxa de emprego muito baixa. O efeito do gasto em educação sobre as variáveis endógenas do modelo deve ser analisado com cautela. Não se deve considerar apenas o resultado isolado da política de gasto do governo em educação. Isso pode gerar 
interpretações equivocadas sobre a importância desse modelo de crescimento econômico.

O que o modelo sugere é que uma política que apenas vise ao aumento da produtividade sem considerar um estímulo similar do lado da demanda - o determinante último do crescimento num modelo kaleckiano - tenderá a demandar menos trabalho por unidade de produção. Se a demanda não aumenta pari passu com o aumento da produtividade, haverá desemprego. É fundamental então que, assim como se atenta para a evolução da produtividade, também se considerem políticas de mudança estrutural destinadas a reduzir a elasticidade da renda das importações $(\pi)$, ou políticas competitivas destinadas a reduzir o mark-up $(z)$, como forma de sustentar uma expansão adequada do lado da demanda.

Em relação à alíquota de tributação sobre o salário ofertado $(\tau)$, há apenas uma diferença entre os resultados encontrados para o parâmetro $g$. A taxa de câmbio pode responder positivamente ou negativamente a um aumento na alíquota de tributação. Se a velocidade de resposta do nível de emprego $(\gamma)$ for maior do que a relação da alíquota de tributação $\gamma>\frac{\tau}{1+\tau}$, haverá um aumento da taxa de câmbio. Nesse caso, lembrando que a receita tributária obtida com $\tau$ será utilizada para o gasto em educação, então o ganho de produtividade compensará os custos do aumento desse imposto sobre o salário ofertado pelas firmas. Caso contrário, $\gamma<\frac{\tau}{1+\tau}$, haverá uma redução da taxa de câmbio e o ganho de produtividade não compensará o aumento na alíquota de imposto, tornando o produto doméstico mais caro em relação ao internacional.

Outro parâmetro importante a ser mencionado é a taxa de crescimento da renda internacional $\left(\hat{Y}^{*}\right)$. Nesse modelo, como ilustrado na Tabela 2, um aumento na taxa de crescimento da renda internacional provoca efeitos positivos sobre o nível de emprego $(\tilde{n})$ e efeitos adversos sobre a taxa de câmbio $(\tilde{q})$ (passando do ponto de equilíbrio $a$ para $b$ ). Esses efeitos são representados na Figura 3. O aumento no emprego $\left(\tilde{n}_{1} \rightarrow \tilde{n}\right)$ se explica pelo maior nível de demanda efetiva proporcionado pelo aumento da renda internacional. Esse aumento no nível de emprego provoca um aumento na participação do salário real na renda doméstica produzida. Isto é, há um aumento nos custos domésticos de produção, em termos de salário, em relação aos custos do resto do mundo. O aumento dos custos de produção domésticos se explicita neste modelo como uma valorização da taxa de câmbio $\left(\tilde{q} \rightarrow \tilde{q}_{1}\right)$. 
Figura 3

Aumento na taxa de crescimento da renda internacional

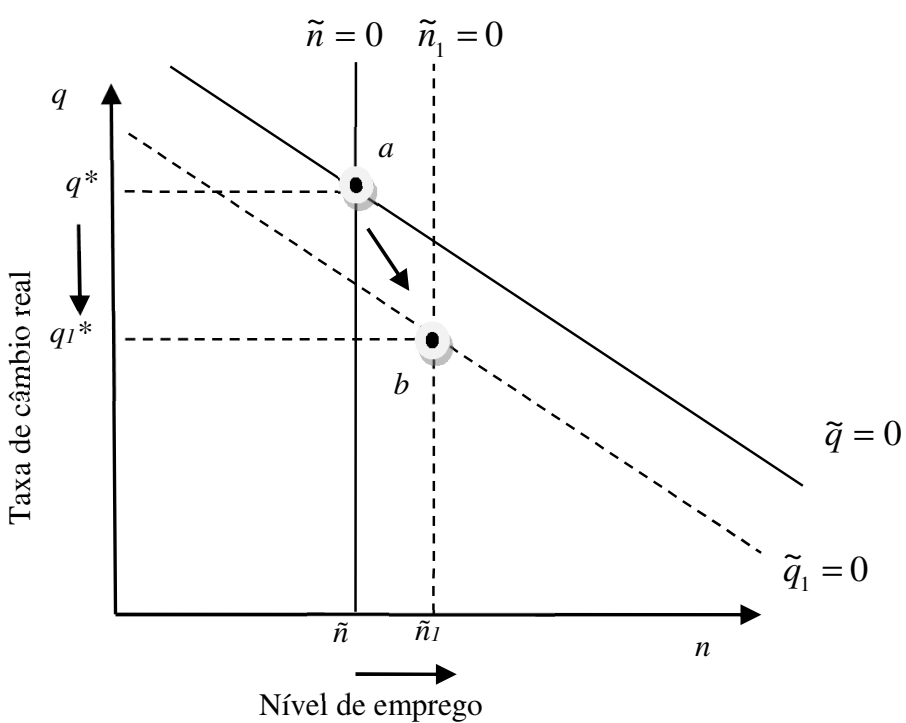

Como observado na Tabela 2, o resultado do aumento na taxa de crescimento da renda internacional $\left(\hat{Y}^{*}\right)$ é positivo sobre a taxa de crescimento da renda doméstica $(\hat{Y})$.Isso é explicado pelo fato de que o crescimento no nível de emprego $(\tilde{n})$ aumenta a arrecadação tributária $(Y \tau)$ e, consequentemente, o volume de gasto público investido em educação $(\mathrm{g})^{17}$, ampliando a acumulação de capital humano, e, por conseguinte, elevando a produtividade do trabalho doméstico. Esse efeito sobre a produtividade é maior do que o efeito de valorização da taxa de câmbio; como resultado, aumenta a taxa de crescimento da renda doméstica $(\hat{Y})$.

Em relação à elasticidade da renda das importações $(\pi)$ e à taxa de crescimento da produtividade no resto do mundo $(\hat{\rho})$, ambos os parâmetros se relacionam negativamente com o nível de emprego e a taxa de crescimento da renda $(\hat{Y})$. Isto é, um aumento na elasticidade da renda das importações $(\pi)$ ou na taxa de crescimento da produtividade no resto do mundo $(\hat{\rho})$ gera efeitos negativos sobre o nível de emprego doméstico e, por conseguinte, sobre a taxa de crescimento da renda. O aumento nesses parâmetros $(\pi$ e $\hat{\rho})$ gera efeitos positivos com relação à taxa de câmbio.

(17) Assim como todos os outros parâmetros, a porcentagem do gasto público (g) e a alíquota de tributação $(\tau)$ se mantêm constantes. Mas como há um aumento da renda doméstica $(Y)$, supondo que a distribuição da renda entre os agentes se mantém na mesma proporção, a parcela da renda doméstica destinada aos capitalistas e aos trabalhadores será maior. 
Em outras palavras, uma redução do crescimento da economia mundial ( $\left.\hat{Y}^{*}\right)$, como a que se visualiza nos próximos anos, tem efeitos negativos em várias áreas: (i) reduz a taxa de emprego; (ii) reduz a taxa de crescimento do país; (iii) aumentando q, há uma piora na participação dos salários na renda. Esse último fator, em conjunto com a redução na taxa de emprego, gera um ambiente no qual os trabalhadores são fortemente prejudicados (via desemprego e via menor participação na renda dos empregados).

O mesmo acontece se houver um aumento na taxa de progresso técnico (exógeno) do resto do mundo. Neste último caso, no entanto, se for assumido (adicionalmente) que a taxa de crescimento da produtividade $(\hat{\rho})$ e a da renda no resto do mundo $\left(\hat{Y}^{*}\right)$ estão associadas, o aumento de $\hat{\rho}$ e de $\hat{Y} *$ pode se compensar e reduzir assim o impacto negativo do maior valor de $\hat{\rho}$.

O efeito da intensidade da resposta das exportações à dinâmica da produtividade interna e externa $(\varepsilon)$ sobre a taxa de câmbio dependerá da seguinte relação entre os parâmetros: se $\hat{Y}^{*}>\rho \pi$, a intensidade da resposta provocará uma queda na taxa de câmbio; já se $\hat{Y}^{*}<\rho \pi$, haverá um aumento na taxa de câmbio. Em relação ao nível de emprego ( $\tilde{n})$ e à taxa de crescimento da renda doméstica ( $\hat{Y})$, se $\hat{Y}^{*}>\rho \pi$, a intensidade da resposta à dinâmica tecnológica interna e externa $(\varepsilon)$ gera efeitos positivos sobre essas duas variáveis endógenas, mas se $\hat{Y}^{*}<\rho \pi$, os efeitos serão adversos sobre o nível de emprego e a taxa de crescimento da renda doméstica. Uma forma de entender intuitivamente essa relação é considerar que a resposta à elasticidade-produtividade das exportações é favorável ao crescimento e ao emprego quando a taxa de crescimento da demanda autônoma mundial supera o efeito combinado (sobre as importações) da expansão da demanda interna e do aumento da produtividade do resto do mundo. Tanto o aumento da renda nacional como o aumento da produtividade do resto do mundo favorecem as importações: se sua influência supera o efeito positivo do aumento da renda do resto do mundo sobre as exportações, então uma elevada resposta das exportações aos diferenciais de produtividade não seria favorável.

Por sua vez, um aumento no mark-up (z) gera um efeito de desvalorização da taxa de câmbio e um efeito adverso sobre o nível de emprego $(\tilde{n})$ e sobre a taxa de crescimento da renda doméstica $(\hat{Y})$. Os efeitos negativos sobre a distribuição e o crescimento do aumento do grau de monopólio, previsto por Blecker (1999), se confirmam no contexto de um modelo dinâmico com acumulação de capital humano. No modelo de Blecker (1989), a piora está associada à menor competitividade e à queda do consumo interno. Nesse modelo, o efeito negativo ocorre pela via da perda de competitividade e pela perda de acumulação de capital humano, esta última associada a um menor nível de emprego. Nesse sentido, uma 
redução do grau de monopólio da economia teria efeitos positivos sobre várias frentes - no aumento da taxa de emprego, nos níveis de equidade e na taxa de crescimento.

Por fim, a velocidade de resposta do nível de emprego $(\gamma)$ relaciona-se inversamente com a taxa de câmbio em decorrência do aumento do custo de produção doméstico (aumento do salário). No entanto, o efeito sobre o nível de emprego e sobre a taxa de crescimento da renda doméstica dependerá dos valores dos seguintes parâmetros: se $z>\psi+q(1-\alpha)$, então o aumento na velocidade de resposta do nível de emprego produzirá um efeito negativo sobre o nível de emprego $(\tilde{n})$, mas se, ao contrário, $z<\psi+q(1-\alpha)$, então haverá um efeito positivo sobre o nível de emprego $(\tilde{n})$ e sobre a taxa de crescimento da renda doméstica $(\hat{Y})$. Assim, dado $\gamma$, valores mais baixos do mark-up contribuem para sustentar o emprego e o crescimento econômico.

Em suma, nesta seção foi feita uma análise de dinâmica comparativa de um modelo kaleckiano de crescimento com acumulação de capital humano e aprendizado. Os exercícios de estática comparativa sugerem que mudanças em alguns parâmetros estimulam, ao mesmo tempo, crescimento e distribuição. Aumentos na renda mundial e redução da elasticidade-renda das importações e do grau de monopólio, em particular, permitem aumentar, ao mesmo tempo, o emprego e o crescimento, e elevar a participação dos trabalhadores na renda nacional. Já aumentos no gasto do governo em educação (g) favorecem o crescimento, mas reduzem a taxa de emprego e pioram a distribuição. Isso ressalta a importância de considerar as políticas de elevação da produtividade do trabalho em conjunto com as políticas que procuram sustentar a demanda efetiva em níveis elevados. Por exemplo, políticas de mudança estrutural que procuram diminuir a elasticidade da renda das importações $(\pi)$ poderiam complementar políticas de investimento em capital humano, para expandir a produtividade sem comprometer a taxa de emprego.

\section{Conclusão}

Neste artigo objetivou-se fazer uma contribuição analítica sobre os fatores que determinam o nível de emprego, a taxa de câmbio e a produtividade do trabalho no contexto de um modelo kaleckiano em que a política fiscal também tem efeitos sobre a produtividade do trabalho. Para tanto, o principal aspecto abordado foi a importância do gasto do governo em educação como instrumento para elevar a produtividade do trabalho e, por conseguinte, acelerar o crescimento de uma economia aberta em desenvolvimento. Essa relação entre gasto público, educação, produtividade e crescimento é analisada no contexto de um modelo kaleckiano em que a taxa de crescimento está sujeita à restrição externa. 
A temática da barganha salarial e da distribuição da renda foi utilizada como marco inicial para incluir a análise do papel do gasto público no desenvolvimento macroeconômico. A política fiscal foi apresentada de outra perspectiva neste trabalho. Apesar de mais impostos tornarem mais intenso o conflito distributivo, o modelo sugere que devem ser vistos seus efeitos a partir de uma perspectiva mais ampla, considerando a evolução da produtividade do trabalho. O gasto do governo em educação é um dos usos dos impostos e, nessa medida, pode elevar a produtividade do trabalho, expandir o produto e - por meio de alguma divisão do ganho obtido entre trabalhadores e empregadores - reduzir as tensões entre as duas classes. $\mathrm{O}$ aumento da produtividade e do produto no tempo pode tornar mais favorável a negociação do conflito distributivo.

Um aspecto importante a ser ressaltado é a relação entre a alíquota de tributação sobre o salário ofertado $(\tau)$ e a taxa de câmbio. Neste trabalho foi suposto que a receita tributária obtida com $\tau$ seria utilizada no gasto em educação. Logo, dependendo da magnitude da velocidade de resposta do nível de emprego ( $($ ) , o ganho de produtividade pode ou não compensar o custo da tributação sobre o salário real ofertado pelas firmas. O resultado da interação desses dois parâmetros $(\gamma$ e $\tau)$ determinará em que direção a taxa de câmbio afetará os custos de produção domésticos.

Nem todos os efeitos do gasto são favoráveis para as duas classes. O aumento do gasto do governo em educação produz efeitos negativos sobre o nível de emprego $(\tilde{n})$. No entanto, esse efeito negativo é mais do que compensado pelo aumento na taxa de crescimento da renda real $(\hat{Y})$. Desse modo, ter-se-ia uma versão dinâmica do paradoxo dos custos. $\mathrm{O}$ efeito positivo gerado pelo acúmulo de capital humano e pelo aumento na produtividade do trabalho compensaria dinamicamente o maior custo associado aos impostos. Também surge do modelo a ideia de que períodos de mais rápida expansão do produto mundial, assim como esforços de mudança estrutural no sentido de reduzir a elasticidade-renda das importações, podem favorecer ao mesmo tempo crescimento, distribuição da renda e elevação da taxa de emprego. É interessante notar que uma política apenas dirigida à educação, se não é acompanhada por políticas de mudança estrutural ou redução do grau de monopólio da economia, poderia elevar a taxa de desemprego. A política industrial e a política de educação deveriam ser implementadas em sincronia, respeitando o grau de intensidade ótimo entre as duas políticas para maximizar o desenvolvimento econômico do país.

Existe uma agenda de pesquisas que poderia ser desenvolvida a partir do modelo sugerido neste artigo. No campo teórico, seria interessante considerar outros tipos de uso dos impostos, por exemplo, para promover exportações ou a mudança estrutural, afetando os parâmetros $\varepsilon$ e $\pi$. O imposto seria destinado a usos alternativos e haveria formas mais desejáveis de distribuição do ponto de vista da 
relação entre crescimento e distribuição. Seria interessante também discutir quais tipos de medidas maximizam a melhora na distribuição da renda, considerando também a situação dos desempregados (que não participam diretamente da barganha salarial), modelando a distribuição total dos trabalhadores (empregados e desempregados). Finalmente, seria interessante realizar testes empíricos para identificar em que medida os efeitos sugeridos pelo modelo têm aderência com os dados - particularmente no que se refere ao vínculo positivo entre gastos em educação e taxas mais altas de crescimento da economia e do salário real.

\section{Referências bibliográficas}

BLECKER, R. International competition, income distribution and economic growth, Cambridge Journal of Economics, v. 13, n. 3, p. 395-412, Sept. 1989.

Kaleckian macro models for open economies. In: DEPREZ, J.; HARVEY, J. T. (Ed.). Foundations of international economics: post Keynesian perspectives. London: Routledge, 1999. p. 116-149.

CARLIN, W.; SOSKICE, D. Macroeconomics and the wage bargain: a modern approach to employment, inflation and the exchange. New York: Oxford University Press, 1990.

Macroeconomics: imperfections, institutions, and policies. New York: Oxford University Press, 2006.

CASSETTI, M. Bargaining power, effective demand and technical progress: a kaleckian model of growth. Cambridge Journal of Economics, v. 27, n. 3, p. 449-464, 2003.

DUTT, A. K. Growth, distribution an uneven development. Cambridge, United Kingdom: Cambridge University Press, 1990.

LAYARD, R.; NICKELL, S.; JACKMAN, R. Macroeconomic performance and the labour market. New York: Oxford University Press, 1991.

LEDESMA, L. Accumulation, innovation and catching-up: an extended cumulative growth model. Cambridge Journal of Economics, v. 26, n. 2, p. 201-216, Mar. 2002.

LEWIS, H. G. Union relative wage effects: a survey. Chicago: University of Chicago Press, 1986.

LUCAS, R. On the mechanics of economic development. Journal of Monetary Economics, v. 22 , n. 1, p. 3-42, 1988.

MANKIW, N. G.; ROMER, D.; WEIL, D. N. A contribution to the empirics of economics growth. Quarterly Journal of Economics, v. 107, n. 2, p. 407-437, May 1992.

MCCOMBIE, J.; THIRLWALL, A. P. Economic growth and the balance of payments constraint. London: St. Martins, 1994.

MCCOMBIE, J. Growth in an international context: a post-Keynesian view. In: DEPREZ, J.; HARVEY, J. T. (Ed.). Foundations of international economics: post Keynesian perspectives. London: Routledge, 1999. 
SEN, A.; DUTT, A. K. Wage bargaining, imperfect competition and the markup: optimizing microfoundations. Economics Letters, v. 48, n. 1, p. 15-20, 1995.

SHONE, R. Economic dynamics: phase diagrams and their economic applications. Cambridge: Cambridge University Press, 1997.

STEINDL, J. Maturity and stagnation in American capitalism. Oxford: Basil Blackwell, 1952.

STEWART, M. B. Relative earnings and individual union membership in the United Kingdom. Economica, v. 50, n. 198, p. 111-126, May 1983. Disponível em: http://www.jstor.org/stable/2554055. Acesso em: 12 maio 2008.

TAKAYAMA, A. Analytical methods in economics. Michigan: The University of Michigan Press, 1993.

THIRLWALL, A. P. The balance of payments constraint as an explanation of international growth rates differences. Banca Nazionale del Lavoro Quarterly Review, p. 45-53, mar. 1979. 